\title{
On the Validity of the Girsanov Transformation Method for Sensitivity Analysis of Stochastic Chemical Reaction Networks
}

\author{
Ting Wang ${ }^{\mathrm{a}}$ and Muruhan Rathinam ${ }^{\mathrm{b}}$ \\ ${ }^{a}$ Department of Mathematical Sciences, University of Delaware, Newark, USA; ${ }^{b}$ Department \\ of Mathematics and Statistics, University of Maryland Baltimore County, Baltimore, USA
}

\author{
ARTICLE HISTORY \\ Compiled November 10, 2020
}

\begin{abstract}
We investigate the validity of the Girsanov Transformation (GT) method for parametric sensitivity analysis of stochastic models of chemical reaction networks. The validity depends on the likelihood ratio process being a martingale and the commutation of a certain derivative with expectation. We derive some exponential integrability conditions which imply both these requirements. We provide further conditions in terms of a reaction network that imply these exponential integrability conditions.
\end{abstract}

\section{KEYWORDS}

Girsanov transformation, sensitivity analysis, chemical reaction networks, exponential integrability

\section{Introduction}

Parametric sensitivity analysis is an essential part of modeling and analysis of dynamical systems. In the context of stochastic dynamical systems the problem that is considered frequently is that of estimating the sensitivity defined by the partial derivative

$$
\left.\frac{\partial}{\partial c}\right|_{c=c^{*}} \mathbb{E} f(X(T, c))
$$

where $c$ is a parameter of interest, $c^{*}$ is its nominal value, $X$ is the stochastic process, $f$ is a scalar function of the state and $T>0$ is a fixed terminal time.

While we focus on the well-stirred stochastic model of chemical kinetics [6], we like to mention that similar models arise in applications that are concerned with populations (nonnegative integer vectors). Due to the high dimensionality of the state space of the Markov process $X$ describing the chemical kinetics, Monte Carlo methods are usually the most viable. Monte Carlo methods of sensitivity analysis for stochastic chemical models can be classified into the finite difference methods (FD) [1, 16], the Girsanov Transformation (GT) method [14], the regularized pathwise derivative (RPD) method [18] and what might be termed the auxiliary path (AP) type methods [8, 9]. When

\footnotetext{
a Email: tingw@udel.edu

bEmail: muruhan@umbc.edu
} 
considering more general applications, one again finds roughly, a similar classification [2]. Among these methods, the FD methods are always biased and the RPD method is biased in the context of chemical kinetics and is not always applicable. The well known GT method is usually widely applicable and is unbiased. The main shortcoming of the GT method is that it has been observed and that it often has large variance and hence less efficient [19]. For an asymptotic analysis of the variance of the GT and related methods, see [19] where a centralized GT method is shown to be more efficient under certain circumstances. The recently introduced auxiliary path type methods are unbiased as well, and provide for an alternative to the GT methods. Nevertheless, due to the ease of implementation, the GT method is of interest.

The validity of the GT method has been studied in the literature under various contexts [7, 13]. We briefly describe the differences and similarities of our work in comparison with these earlier works.

First we note that the analysis in [7, 13] which provides sufficient conditions under which the GT method is applicable is formulated in terms of a discrete time Markov chain. On the other hand, the analysis in our paper is formulated in terms of a continuous time Markov chain and provides a self-contained description of the GT method based on [3]. While in principle there is a precise relationship between the two approaches, this relationship is rather cumbersome. In particular, the fact that a fixed final time $T$ in our continuous time setting translates to a random stopping time $N$ in the discrete time approach results in added assumptions on the nature of the dependence of $N$ on parameters: see for instance the assumption A2(iii) in [7] which assumes $L^{1}$-differentiability (with respect to the parameter) of the ratios of the values of $N$ at a general parameter value to that at a reference parameter value. There is no such assumption in our analysis.

Secondly, our analysis is focused on a limited, albeit practically important class of continuous time Markov chains, namely, stochastic reaction networks. This imposes extra structure, resulting in a simpler set of sufficient conditions.

The previous works [7, 13] as well as our work, all result in a form of exponential integrability requirement as part of the set of sufficient conditions ensuring the validity of the GT method. The works [7, 13] require exponential integrability of the event count, while our paper provides one of two alternative requirements: (23) or (26). One requires exponential integrability of event count while the other requires exponential integrability of the intensity (propensity) function. This second alternative condition doesn't appear to have a counter part in [7, 13]. Verification of either one of these is adequate under the assumption that the required sensitivity exists, which was shown to follow under milder conditions [8].

The verification of either one of these exponential integrability conditions is not so straightforward. Bearing this in mind, in the context of stochastic chemical reaction networks, we derive some sufficient conditions for the validity of GT method that can be verified via an algorithm given the network parameters. We also illustrate via examples how our sufficient conditions may be verified for a rich class of reaction networks using a probabilistic coupling argument.

\subsection{Stochastic chemical kinetics}

We describe the stochastic model of well-stirred chemical reactions involving $n$ molecular species undergoing $m$ reaction channels [6]. In this model, the molecular copy number vector $X(t) \in \mathbb{Z}_{+}^{n}(t \geq 0)$ is considered as a Markov process in continuous 
time. Occurrence of $j$ th reaction leads to a change of $X(t)$ by $\nu_{j} \in \mathbb{Z}^{n}$ for $j=1, \ldots, m$ where the $\nu_{j}$ are known as the stoichiometric vectors. We denote by $R_{j}(t)$ the counting process which counts the number of occurrences of reaction channel $j$ during $(0, t]$, for $j=1, \ldots, m$. The probabilistic rate of occurrence of reaction $j$, is given by the intensity function or propensity function $a_{j}(x)$ which is defined such that conditioned on $X(t)=x$, the probability that $X(t+h)=x+\nu_{j}$ is $a_{j}(x) h+o(h)$ as $h \rightarrow 0+$, and the probability that $X(t+h)=x$ is $1-\sum_{j=1}^{m} a_{j}(x) h+o(h)$ as $h \rightarrow 0+$.

The propensity function, in addition to the state $x$, potentially depends on other factors such as the temperature and system volume and this dependence is captured by a set of parameters which are non-random and constant in time. In particular, in the stochastic form of mass action case, the propensity function is of the product form

$$
a_{j}(x, c)=c_{j} b_{j}(x)
$$

where $c_{j}>0$ is a parameter independent of $x$ and $b_{j}(x)$ is a (multivariate) polynomial in $x$ [6]. While our final results in this paper assume the product form (11) (but not necessarily the mass action form), we shall keep our derivations as general as possible until the final steps.

It is possible to represent the processes for different parameter values $c$ in the same sample space $(\Omega, \mathcal{F}, \mathbb{P})$ (hence the notation $X(t, c)$ and $R(t, c)$ ) via the random time change representation [5]

$$
X(t, c)=x_{0}+\sum_{j=1}^{m} \nu_{j} Y_{j}\left(\int_{0}^{t} a_{j}(X(s, c), c) d s\right),
$$

where $Y_{1}, \ldots, Y_{m}$ are independent unit rate Poisson processes carried by $(\Omega, \mathcal{F}, \mathbb{P})$ and $x_{0} \in \mathbb{Z}_{+}^{n}$ is the initial state. The reaction count processes $R_{j}$ are then given by

$$
R_{j}(t, c)=Y_{j}\left(\int_{0}^{t} a_{j}(X(s, c), c) d s\right) \quad j=1, \ldots, m
$$

We also note the relationship

$$
X(t, c)=x_{0}+\sum_{j=1}^{m} \nu_{j} R_{j}(t, c) .
$$

We note that the processes $X(t, c)$ and $R(t, c)$ are cadlag. We shall also assume that $X(t, c)$ is non-explosive for each $c$, that is, $R_{j}(t, c)<\infty$ for each $t \geq 0$, each $c$ and $j=1, \ldots, m$. For sake of readability, throughout the paper we suppress the dependence of $X$ and $R$ on $\omega \in \Omega$ except when necessary.

Without loss of generality we shall focus on estimation of sensitivity with respect to one scalar parameter $c$ and we assume that it corresponds to the first reaction channel, so that $c=c_{1}$ and thus $a_{1}(x, c)=c b_{1}(x)$ under the product form. Let $c^{*}>0$ be a nominal parameter value and $T>0$ be some terminal time. Given a function $f: \mathbb{Z}_{+}^{n} \rightarrow \mathbb{R}$, we are interested estimating the sensitivity defined by

$$
\left.\frac{\partial}{\partial c}\right|_{c=c^{*}} \mathbb{E} f(X(T, c)) .
$$


Throughout this paper, we assume that the sensitivity exists. We refer the reader to [8] for some sufficient conditions that guarantee the existence of the sensitivity.

\subsection{The Girsanov transformation method}

One of the commonly used sensitivity estimation methods is the Girsanov transformation (GT) method which is also known as the likelihood ratio (LR) method in literature. We first describe the basics of this approach and then furnish details in the context of chemical kinetics. We consider a nominal parameter value $c^{*}$ and an open interval $I_{c^{*}}=\left(c^{*}-\epsilon, c^{*}+\epsilon\right)$. As mentioned before, we assume that the processes $X(t, c)$ and $R(t, c)$ for $c \in I_{c^{*}}$ are all carried by a common probability space $(\Omega, \mathcal{F}, \mathbb{P})$. Let us denote by $\left\{\mathcal{F}_{t}\right\}_{t}$ the filtration generated by $X\left(t, c^{*}\right)$ and $R\left(t, c^{*}\right)$. (We remark that if we assume that the $\nu_{j}$ are all distinct then $\left\{\mathcal{F}_{t}\right\}_{t}$ will be generated by $X\left(t, c^{*}\right)$ alone).

The GT method involves defining for each $c \in I_{c^{*}}$ a probability measure $\mathbb{P}_{c}$ on $(\Omega, \mathcal{F})$ which satisfies the following condition.

Condition 1: For each $c \in I_{c^{*}}$, (i) $\mathbb{P}_{c}$ is absolutely continuous with respect to $\mathbb{P}$, (ii) $\mathbb{P}_{c^{*}}=\mathbb{P}$ and (iii) for every bounded function $f: \mathbb{Z}_{+}^{n} \rightarrow \mathbb{R}$

$$
\mathbb{E} f(X(T, c))=\mathbb{E}_{c} f\left(X\left(T, c^{*}\right)\right),
$$

where $\mathbb{E}$ is the expectation with respect to $\mathbb{P}$ and $\mathbb{E}_{c}$ is the expectation with respect to $\mathbb{P}_{c}$.

Suppose such a family of probability measures $\mathbb{P}_{c}$ satisfying Condition 1 exist. Let us denote by $L(c, t)$ the Radon-Nykodim derivative

$$
L(t, c)=\left.\frac{d \mathbb{P}_{c}}{d \mathbb{P}}\right|_{\mathcal{F}_{t}}
$$

Due to (5), the sensitivity can be written as

$$
\left.\frac{\partial}{\partial c}\right|_{c=c^{*}} \mathbb{E} f(X(T, c))=\left.\frac{\partial}{\partial c}\right|_{c=c^{*}} \mathbb{E}_{c} f\left(X\left(T, c^{*}\right)\right)=\left.\frac{\partial}{\partial c}\right|_{c=c^{*}} \mathbb{E}\left[f\left(X\left(T, c^{*}\right)\right) L(T, c)\right] .
$$

Condition 2: Suppose that the derivative

$$
Z\left(t, c^{*}\right)=\left.\frac{\partial}{\partial c}\right|_{c=c^{*}} L(t, c)
$$

exists almost surely with respect to $\mathbb{P}_{c^{*}}$ and that the following commutation of derivative and expectation holds:

$$
\mathbb{E}\left(\left.\frac{\partial}{\partial c}\right|_{c=c^{*}} f\left(X\left(T, c^{*}\right)\right) L(T, c)\right)=\left.\frac{\partial}{\partial c}\right|_{c=c^{*}} \mathbb{E} f\left(X\left(T, c^{*}\right)\right) L(T, c) .
$$

This leads to the formula

$$
\left.\frac{\partial}{\partial c}\right|_{c=c^{*}} \mathbb{E} f(X(T, c))=\mathbb{E}\left[f\left(X\left(T, c^{*}\right)\right) Z\left(T, c^{*}\right)\right]
$$


Thus if Conditions 1 and 2 are satisfied, the required sensitivity equals the expected value of the random variable $f\left(X\left(T, c^{*}\right)\right) Z\left(T, c^{*}\right)$ and hence can be estimated via iid sample estimation. Thus $\hat{s}_{N}$ given by

$$
\hat{s}_{N}=\frac{1}{N} \sum_{i=1}^{N} f\left(X^{(i)}\left(T, c^{*}\right)\right) Z^{(i)}\left(T, c^{*}\right),
$$

where $\left(X^{(i)}\left(T, c^{*}\right), Z^{(i)}\left(T, c^{*}\right)\right)$ for $i=1, \ldots, N$ are iid pairs of samples, is the GT estimator for a sample size of $N$. We note that the simulation is carried out with respect to the probability measure $\mathbb{P}=\mathbb{P}_{c^{*}}$.

The GT estimator is unbiased but often has large variance unless $N$ is very large [19]. Nevertheless, due to its simplicity, GT has been widely applied for sensitivity analysis in numerous areas such as chemical kinetics and operations research.

While the GT method is widely used, we are not aware of theoretical studies on the validity of GT method. In particular, in the area of stochastic reaction networks, no sufficient conditions have been provided to justify the method. Therefore, we believe a theoretical analysis of the method could provide a guideline about the applicability of GT to certain types of problems. In this paper, we aim to provide sufficient conditions that ensure Conditions 1 and 2 stated above.

\section{The validity of change of measure}

\subsection{Change of intensity}

We explore some sufficient conditions that guarantee Condition 1 for the change of measure. Our exposition here is based on the change of intensity theory in Section VI 2 of [3]. We start with the probability space $(\Omega, \mathcal{F}, \mathbb{P})$ on which the processes $X(t, c)$ and $R(t, c)$ are defined for $c \in I_{c^{*}}=\left(c^{*}-\epsilon, c^{*}+\epsilon\right)$. As before, we denote by $\mathcal{F}_{t}$ the filtration generated by $X\left(t, c^{*}\right)$ and $R\left(t, c^{*}\right)$. By definition, the counting process $R_{j}\left(t, c^{*}\right)$ has the $\left(\mathbb{P}, \mathcal{F}_{t}\right)$ predictable intensity $a_{j}\left(X\left(t-, c^{*}\right), c^{*}\right)$. Now for any $c \in I_{c^{*}}$, we want to explicitly construct a probability measure $\mathbb{P}_{c}$ on $(\Omega, \mathcal{F})$ such that $R\left(t, c^{*}\right)$ admits the $\left(\mathbb{P}_{c}, \mathcal{F}_{t}\right)$ predictable intensity $a_{j}\left(X\left(t-, c^{*}\right), c\right)$. This is accomplished by defining the likelihood ratio process $L(t, c)$ which under the right conditions will serve as the Radon-Nykodim derivative.

We first define an auxiliary ( $m$ dimensional) process $\mu(t, c)$ under a certain assumption on the propensity functions as follows. Given an arbitrary $c \in I_{c^{*}}$, we assume that for all $x \in \mathbb{Z}_{+}^{n}$,

$$
a_{j}\left(x, c^{*}\right)=0 \text { if and only if } a_{j}(x, c)=0, \quad j=1,2, \cdots, m .
$$

We note that this is assumption holds in particular when the propensity functions are of the product form $a_{j}(x, c)=c_{j} b_{j}(x)$. Now, based on this assumption, the following process is well-defined ( $c^{*}$ is fixed). For each $c \in I_{c^{*}}$, we define

$$
\mu_{j}(t, c)=\frac{a_{j}\left(X\left(t-, c^{*}\right), c\right)}{a_{j}\left(X\left(t-, c^{*}\right), c^{*}\right)}, \quad j=1, \cdots, m
$$

In the case that $a_{j}\left(X\left(t-, c^{*}\right), c^{*}\right)=0$, by assumption we have $a_{j}\left(X\left(t-, c^{*}\right), c\right)=0$ as well, so we can simply define $\mu_{j}(t, c)$ to be any strictly positive constant. We note 
that $\mu_{j}(t, c)$ is $\mathcal{F}_{t}$-predictable by its left continuity, and moreover for each $t \geq 0$, we have $0<\mu_{j}(t, c)<\infty$ almost surely. We shall make the extra assumption that $\mu_{j}(t, c)$ is bounded almost surely for each $c \in I_{c^{*}}$. In the case of the product form of propensity functions with $c=c_{1}$, we note that the boundedness assumption holds since $\mu_{1}(t, c)=c / c^{*}$ and $\mu_{j}(t, c)=1$ for $j=2, \ldots, m$, that is the process $\mu(t, c)$ is a deterministic and constant in $t$.

Next, following [3] , we explicitly define the likelihood ratio process $L(t, c)$ as follows

$$
L(t, c)=\prod_{j=1}^{m}\left(\left(\prod_{n=1}^{R_{j}\left(t, c^{*}\right)} \mu_{j}\left(T_{j}^{n}, c\right)\right) \exp \left(\int_{0}^{t}\left(1-\mu_{j}(s, c)\right) a_{j}\left(X\left(s, c^{*}\right), c^{*}\right) d s\right)\right)
$$

where $T_{j}^{n}$ is the $n$-th jump time of $R_{j}\left(t, c^{*}\right)$. By convention, we take the product $\prod_{n=1}^{R_{j}\left(t, c^{*}\right)}$ to be 1 if $R_{j}\left(t, c^{*}\right)=0$. We remark that due to our non-explosivity assumption $R_{j}\left(t, c^{*}\right)$ is finite almost surely for each $t$ and thus $L(t, c)$ is well defined and satisfies $0<L(t, c)<\infty$ for each $t \geq 0$.

It can be shown that $L$ defined above is the solution of the equation [3]

$$
L(t, c)=1+\sum_{j=1}^{m} \int_{(0, t]} L(s-, c)\left(\mu_{j}(s, c)-1\right) d M_{j}\left(s, c^{*}\right)
$$

where

$$
M_{j}\left(t, c^{*}\right)=R_{j}\left(t, c^{*}\right)-\int_{0}^{t} a_{j}\left(X\left(s, c^{*}\right), c^{*}\right) d s .
$$

From the non-explosivity assumption, we see that for all $t \geq 0$ and $j=1, \cdots, m$,

$$
\int_{0}^{t} a_{j}\left(X\left(s, c^{*}\right), c^{*}\right) d s<\infty, \mathbb{P} \quad \text { a.s. }
$$

and hence $M\left(t, c^{*}\right)$ is an $m$-dimensional local martingale [3]. We summarize some key results from [3] as a lemmas.

Lemma 2.1. (Bremaud [3], Section VI, Theorem T2) Under the non-explosivity assumption (with respect to $\mathbb{P}$ ), for each $c \in I_{c^{*}}, L(t, c)$ is a $\left(\mathbb{P}, \mathcal{F}_{t}\right)$ nonnegative local martingale and hence $a\left(\mathbb{P}, \mathcal{F}_{t}\right)$ supermartingale.

Lemma 2.2. (Bremaud [3], Section VI, Theorem T3) Suppose that $\mathbb{E} L(T, c)=$ 1. Then $L(t, c)$ is a $\left(\mathbb{P}, \mathcal{F}_{t}\right)$ martingale over $[0, T]$. Moreover, defining the probability measures $\mathbb{P}_{c}$ by the condition

$$
\frac{d \mathbb{P}_{c}}{d \mathbb{P}}=L(t, c),
$$

it follows that over $[0, T], R_{j}\left(t, c^{*}\right)$ has the $\left(\mathbb{P}_{c}, \mathcal{F}_{t}\right)$-intensity

$$
a_{j}\left(X\left(t-, c^{*}\right), c\right)=\mu_{j}(t, c) a_{j}\left(X\left(t-, c^{*}\right), c^{*}\right) .
$$


Corollary 2.3. Under the conditions of Lemma 2.2, for each bounded measurable function $f: \mathbb{Z}_{+}^{n} \rightarrow \mathbb{R}$ and each $c \in I_{c^{*}}$

$$
\mathbb{E} f(X(T, c))=\mathbb{E}_{c} f\left(X\left(T, c^{*}\right)\right) .
$$

In other words, the $\mathbb{P}_{c}$ law of $X\left(t, c^{*}\right)$ is the same as the $\mathbb{P}$ law of $X(t, c)$.

Proof. The law of a Markov process is uniquely determined by the Kolmogorov's forward equation. The form of the forward equation depends on the state dependence of the intensities of the jump event. Since the $\mathbb{P}$ intensity of $R_{j}(t, c)$ is $a_{j}(X(t-, c), c)$ and the $\mathbb{P}_{c}$ intensity of $R_{j}\left(t, c^{*}\right)$ is $a_{j}\left(X\left(t-, c^{*}\right), c\right)$, the state dependence are the same for both: $a_{j}(x, c)$. Thus the Kolmogorov's forward equations for $p_{1}(x, t)=\mathbb{P}(X(t, c)=x)$ and $p_{2}(x, t)=\mathbb{P}_{c}\left(X\left(t, c^{*}\right)=x\right)$ are identical:

$$
\frac{d p_{i}(x, t)}{d t}=\sum_{j=1}^{m}\left(p_{i}\left(x-\nu_{j}, t\right) a_{j}\left(x-\nu_{j}, c\right)-p_{i}(x, t) a_{j}(x, c)\right), \quad x \in \mathbb{Z}_{+}^{n}, \quad i=1,2 .
$$

In summary, the condition $\mathbb{E}(L(T, c))=1$ in Lemma 2.2 ensures the validity of the change of measure. In the next section we provide some sufficient conditions for it to hold.

\subsection{Novikov type condition}

In this section, we provide a Novikov type sufficient condition to ensure that $L(t, c)$ is a martingale over $[0, T]$ or equivalently $\mathbb{E}(L(T, c))=1$. Under the product form of propensities $a_{j}(x, c)=c_{j} b_{j}(x)$, and without loss of generality, taking $c=c_{1}$, the likelihood ratio $L(t, c)$ can be written as

$$
L(t, c)=\left(\frac{c}{c^{*}}\right)^{R_{1}\left(t, c^{*}\right)} \exp \left(\int_{0}^{t}\left(c^{*}-c\right) b_{1}\left(X\left(s, c^{*}\right)\right) d s\right) .
$$

We make the following useful observation. We have

$$
L(t, c) \leq\left(\frac{c}{c^{*}}\right)^{R_{1}\left(t, c^{*}\right)}
$$

for any $c \in I_{c^{*}}^{+}=\left[c^{*}, c^{*}+\epsilon\right)$ and

$$
L(t, c) \leq \exp \left(\int_{0}^{t}\left(c^{*}-c\right) b_{1}\left(X\left(s, c^{*}\right)\right) d s\right)
$$

for any $c \in I_{c^{*}}^{-}=\left(c^{*}-\epsilon, c^{*}\right]$. This simple observation turns out to be useful for our analysis.

Theorem 2.4. Given $c \in I_{c^{*}}^{+}$, suppose that

$$
\mathbb{E}\left[\left(\frac{c}{c^{*}}\right)^{R_{1}\left(T, c^{*}\right)}\right]<\infty
$$


then $L(t, c)$ is a $\left(\mathbb{P}, \mathcal{F}_{t}\right)$ martingale over $[0, T]$.

Proof. By Lemma $2.1 L(t, c)$ is a local martingale. Thus there exists a sequence $\left(\sigma_{n}\right)$ of increasing stopping times with $\sigma_{n} \uparrow \infty$ such that $L\left(t \wedge \sigma_{n}, c\right)$ is a $\left(\mathbb{P}, \mathcal{F}_{t}\right)$ martingale for each $n$. Define the stopping times

$$
\tau_{n}=\inf \left\{t \geq 0 \mid R_{1}\left(t, c^{*}\right) \geq n\right\} .
$$

By the non-explosivity assumption, $\tau_{n} \uparrow \infty$. We define the stopped processes

$$
L_{n}(t, c)=L\left(t \wedge \sigma_{n} \wedge \tau_{n}, c\right) .
$$

Now for each $n$, as a stopped martingale, $L_{n}(t, c)$ is a $\mathcal{F}_{t}$ martingale and hence $\mathbb{E} L_{n}(T, c)=1$. By the estimates in (17),

$$
L_{n}(T, c) \leq\left(\frac{c}{c^{*}}\right)^{R_{1}\left(T \wedge \sigma_{n} \wedge \tau_{n}, c^{*}\right)} \leq\left(\frac{c}{c^{*}}\right)^{R_{1}\left(T, c^{*}\right)} .
$$

Hence, the integrability condition (19) implies that $\mathbb{E} L(T, c)=1$ by the dominated convergence theorem and therefore $L(t, c)$ is a martingale over $[0, T]$.

Similar result can be reproduced for the case $c \in I_{c^{*}}^{-}$using the estimates (18).

Theorem 2.5. Given $c \in I_{c^{*}}^{-}$, suppose that

$$
\mathbb{E}\left[\exp \left(\left(c^{*}-c\right) \int_{0}^{T} b_{1}\left(X\left(s, c^{*}\right)\right) d s\right)\right]<\infty,
$$

then $L(t, c)$ is a $\left(\mathbb{P}, \mathcal{F}_{t}\right)$ martingale over $[0, T]$.

Proof. Define the stopping times $\tau_{n}$ by

$$
\tau_{n}=\inf \left\{t \geq 0: \int_{0}^{t} b_{1}\left(X\left(s, c^{*}\right)\right) d s \geq n\right\} .
$$

The rest of the proof is similar to that of Theorem 2.4.

One can get rid of the time integral by verifying the following stronger condition.

Corollary 2.6. If there exists $\epsilon>0$ such that

$$
\sup _{s \leq T} \mathbb{E}\left[e^{\epsilon T b_{1}\left(X\left(s, c^{*}\right)\right)}\right]<\infty
$$

then $L(t, c)$ is a $\left(\mathbb{P}, \mathcal{F}_{t}\right)$ martingale over $[0, T]$ for any $c \in I_{c^{*}}^{-}=\left(c^{*}-\epsilon, c^{*}\right)$.

Proof. Rearranging the right hand side of (20) and applying Jensen's inequality to the time average integral inside the bracket, we obtain

$$
\mathbb{E}\left[\exp \left(\frac{1}{T} \int_{0}^{T}\left(c^{*}-c\right) T b_{1}\left(X\left(s, c^{*}\right)\right) d s\right)\right] \leq \mathbb{E}\left[\frac{1}{T} \int_{0}^{T} e^{\left(c^{*}-c\right) T b_{1}\left(X\left(s, c^{*}\right)\right)} d s\right]
$$


By Fubini's Theorem we can commute the time integral and the expectation and hence it suffices to show that

$$
\sup _{s \leq T} \mathbb{E}\left[e^{\left(\epsilon T b_{1}\left(X\left(s, c^{*}\right)\right)\right)}\right]
$$

is finite for $\epsilon$ satisfying $\epsilon>c^{*}-c$.

\section{Differentiation inside the integral}

In this section, we provide a sufficient condition for the commutation (7) of Condition 2 via the use of Theorem A.1 in appendix. Referring to Theorem A.1, we take $G$ to be

$$
G(c)=f\left(X\left(T, c^{*}\right)\right) L(T, c) .
$$

We shall assume the product form with $c=c_{1}$. Then it follows at once from (11) that

$$
\frac{\partial}{\partial c} \ln L(T, c)=\frac{1}{c} R_{1}\left(T, c^{*}\right)-\int_{0}^{T} b_{1}\left(X\left(s, c^{*}\right)\right) d s,
$$

hence

$$
\frac{\partial}{\partial c} L(T, c)=L(T, c)\left(\frac{1}{c} R_{1}\left(T, c^{*}\right)-\int_{0}^{T} b_{1}\left(X\left(s, c^{*}\right)\right) d s\right)=\frac{1}{c} L(T, c) M_{1}\left(T, c^{*}\right) .
$$

Then a Lipschitz constant $K(\omega)$ (independent of $c$ ) for $G$ on the interval $I_{c^{*}}$ is

$$
K=\left|f\left(X\left(T, c^{*}\right)\right) M_{1}\left(T, c^{*}\right)\right| \sup _{c \in I_{c^{*}}} \frac{1}{c} L(T, c) .
$$

We first consider $c \in I_{c^{*}}^{+}$(the right hand sensitivity), in which case we have

$$
L(T, c) \leq\left(\frac{c}{c^{*}}\right)^{R_{1}\left(T, c^{*}\right)} \leq\left(\frac{c^{*}+\epsilon}{c^{*}}\right)^{R_{1}\left(T, c^{*}\right)} .
$$

Hence in order to justify the integrability of $K$, it suffices to show that

$$
f\left(X\left(T, c^{*}\right)\right) M_{1}\left(T, c^{*}\right)\left(\frac{c^{*}+\epsilon}{c^{*}}\right)^{R_{1}\left(T, c^{*}\right)}
$$

is integrable. We also note that we may shrink the interval $I_{c^{*}}=\left(c^{*}-\epsilon, c^{*}+\epsilon\right)$ to be as small as we wish.

Theorem 3.1. Assuming the product form (11) with $c=c_{1}$, suppose the following conditions are satisfied:

- $\mathbb{E}\left[\left|f\left(X\left(T, c^{*}\right)\right)\right|^{3}\right]<\infty$; 
- there exists $\epsilon>0$ such that,

$$
\mathbb{E}\left[\left(\frac{c^{*}+\epsilon}{c^{*}}\right)^{R_{1}\left(T, c^{*}\right)}\right]<\infty .
$$

Then

$$
\lim _{c \rightarrow c^{*+}} \frac{\mathbb{E} f(X(T, c))-\mathbb{E} f\left(X\left(T, c^{*}\right)\right)}{c-c^{*}}=\mathbb{E}\left[f\left(X\left(T, c^{*}\right)\right) \lim _{c \rightarrow c^{*+}} \frac{L(T, c)-L\left(T, c^{*}\right)}{c-c^{*}}\right] .
$$

Proof. First we note that by Theorem 2.4, (23) implies the validity of the change of measure for $c \in I_{c^{*}}^{+}$.

Now we need to verify the limit using Theorem A.1. Using the inequality $3 a b c \leq$ $a^{3}+b^{3}+c^{3}$, we can separate the terms and provide the following sufficient conditions,

$$
\begin{aligned}
& \mathbb{E}\left[\left|f\left(X\left(T, c^{*}\right)\right)\right|^{3}\right]<\infty, \\
& \mathbb{E}\left[\left|M_{1}\left(T, c^{*}\right)\right|^{3}\right]<\infty, \\
& \mathbb{E}\left[\left(\frac{c^{*}+\epsilon}{c^{*}}\right)^{3 R_{1}\left(T, c^{*}\right)}\right]<\infty .
\end{aligned}
$$

It is sufficient to show that the third condition implies the second condition. Since the quadratic variation of the local martingale $M_{1}\left(t, c^{*}\right)$ is $R_{1}\left(t, c^{*}\right)$, by the BurkholderDavis-Gundy (BDG) inequality [15],

$$
\mathbb{E}\left(\left|M_{1}\left(T, c^{*}\right)\right|^{4}\right) \leq C \mathbb{E}\left[R_{1}\left(T, c^{*}\right)^{2}\right] .
$$

for some constant $C$. It is obvious that the right hand side is integrable given the second condition. Since $\left|M_{1}\left(T, c^{*}\right)\right|^{4}$ is integrable the result follows.

Similarly, for the left hand side sensitivity, we have

$$
L(T, c) \leq \exp \left(\int_{0}^{T}\left(c^{*}-c\right) b_{1}\left(X\left(s, c^{*}\right)\right) d s\right) \leq \exp \left(\int_{0}^{T} \epsilon b_{1}\left(X\left(s, c^{*}\right)\right) d s\right)
$$

for $c \in I_{c^{*}}^{-}$. Hence, the Lipschitz constant is proportional to

$$
\left|f\left(X\left(T, c^{*}\right)\right) M_{1}\left(T, c^{*}\right)\right| \exp \left(\int_{0}^{T} \epsilon b_{1}\left(X\left(s, c^{*}\right)\right) d s\right) .
$$

It boils down to verifying the following three integrability conditions,

$$
\begin{aligned}
& \mathbb{E}\left[\left|f\left(X\left(T, c^{*}\right)\right)\right|^{3}\right]<\infty, \\
& \mathbb{E}\left[\left|M_{1}\left(T, c^{*}\right)\right|^{3}\right]<\infty, \\
& \mathbb{E}\left[\exp \left(\int_{0}^{T} 3 \epsilon b_{1}\left(X\left(s, c^{*}\right)\right) d s\right)\right]<\infty .
\end{aligned}
$$

We have the following result concerning the left hand side sensitivity. 
Theorem 3.2. Assuming the product form (1) with $c=c_{1}$, suppose further that

- $\mathbb{E}\left[\left|f\left(X\left(T, c^{*}\right)\right)\right|^{3}\right]<\infty$;

- there exists $\epsilon>0$ such that,

$$
\mathbb{E}\left[\exp \left(\int_{0}^{T} \epsilon b_{1}\left(X\left(s, c^{*}\right)\right) d s\right)\right]<\infty
$$

Then

$$
\lim _{c \rightarrow c^{*-}} \frac{\mathbb{E} f(X(T, c))-\mathbb{E} f\left(X\left(T, c^{*}\right)\right)}{c-c^{*}}=\mathbb{E}\left[f\left(X\left(T, c^{*}\right)\right) \lim _{c \rightarrow c^{*-}} \frac{L(T, c)-L\left(T, c^{*}\right)}{c-c^{*}}\right] .
$$

Proof. We only need to show the second integrability condition in (25) holds. Note that $M_{1}\left(t, c^{*}\right)$ in the second term is a local martingale, we apply the (BDG) inequality such that

$$
\mathbb{E}\left\{\left|M_{1}\left(T, c^{*}\right)\right|^{4}\right\} \leq C \mathbb{E}\left[R_{1}\left(T, c^{*}\right)^{2}\right]
$$

for some constant $C$. Hence, it is sufficient to verify that $\mathbb{E}\left[R_{1}\left(T, c^{*}\right)^{2}\right]<\infty$. Applying the BDG inequality again to $\mathbb{E}\left[M_{1}\left(T, c^{*}\right)^{2}\right]$, there exists some constant $\bar{C}$ such that

$$
\mathbb{E}\left[M_{1}\left(T, c^{*}\right)^{2}\right] \leq \bar{C} \mathbb{E}\left[R_{1}\left(T, c^{*}\right)\right]=\bar{C} \mathbb{E}\left[\int_{0}^{T} a_{1}\left(X\left(s, c^{*}\right), c^{*}\right) d s\right]<\infty .
$$

Owing to the simple inequality $(a+b)^{2} \leq 2\left(a^{2}+b^{2}\right)$,

$$
\mathbb{E}\left[R_{1}\left(T, c^{*}\right)^{2}\right] \leq 2 \mathbb{E}\left[\left(\int_{0}^{T} a_{1}\left(X\left(s, c^{*}\right), c^{*}\right) d s\right)^{2}\right]+2 \mathbb{E}\left[M_{1}\left(T, c^{*}\right)^{2}\right]<\infty .
$$

Remark 1. We note that the conditions of Theorem 3.1 guarantee the existence of the right hand derivative (sensitivity) and that the GT method would provide an unbiased estimator of it. Likewise for Theorem 3.2. However, the conditions of these theorems include the restrictive exponential integrability conditions (23) and (26). We do know from [8] that the existence of sensitivity can be guaranteed under milder conditions. Thus, if we assume the existence of sensitivity at $c^{*}$, then verification of either the conditions of Theorem 3.1 or those of Theorem 3.2 can guarantee the validity of the GT method. This will be our focus in the next section.

\section{Sufficient conditions in terms of the network}

The conditions of of Theorems 3.1 and 3.2 are not directly stated in terms of a chemical reaction network. A chemical reaction network is characterized by the stoichiometric matrix $\nu$ (whose columns are the vectors $\nu_{j}$ ) and the propensity functions $a_{j}(x, c)$ which we assume to be of the product form (1). 
We shall focus on the case that $f: \mathbb{Z}_{+}^{n} \rightarrow \mathbb{R}$ is of polynomial growth. This may be stated by the condition that there exists $C>0$ and $r \in \mathbb{Z}_{+}$such that

$$
|f(x)| \leq C\left(1+\|x\|^{r}\right) \quad \forall x \in \mathbb{Z}_{+}^{n} .
$$

In this case, there exist multiple results in the literature that guarantee the condition that $\mid f\left(\left.X\left(T, c^{*}\right)\right|^{3}\right.$ is integrable [4, 10, 17].

On the other hand, the exponential integrability conditions (23) or (26) are harder to satisfy. When $b_{1}(x)$ is linear, the condition (21) is implied by the conditions for the uniform light-tailedness property presented in [10], and since (21) implies (26), this provides a sufficient condition for the validity of the GT method. However, the uniform light-tailedness property presented in [10] may be too stringent as it is concerned with the supremum over the infinite time horizon $[0, \infty)$.

Remark 2. When the reaction network consists only of reactions of the simple monomolecular form $S_{i} \rightarrow S_{j}$ or $\emptyset \rightarrow S_{i}$ or $S_{i} \rightarrow \emptyset$ with the stochastic mass action form of propensities [6], then the species populations can be shown to be sums of multinomial and Poisson random variables [11]. Since Poisson and multinomial random variables $X$ satisfy exponential integrability $\left(\mathbb{E}\left(e^{\epsilon|X|}\right)<\infty\right.$ for each $\left.\epsilon>0\right)$, and moreover, since the propensities are linear, (21) and hence (26) may be verified.

Given a fixed initial state $x_{0} \in \mathbb{Z}_{+}^{n}$ let $\mathcal{S}_{x_{0}} \subset \mathbb{Z}_{+}^{n}$ denote the set of all states that can be reached by the process starting at $x_{0}$. Thus $\mathcal{S}_{x_{0}}$ is the effective state space of the process $X(t, c)$ and it may be finite or infinite. We remark that if $\mathcal{S}_{x_{0}}$ is finite, then the validity of the GT method follows trivially. As a consequence, the case of interest to us is when $\mathcal{S}_{x_{0}}$ is infinite.

In the rest of this section, we present some sufficient conditions that imply (23) (in the context of reaction networks). Our main idea is to focus on some reactions $R_{j}$ which may be easily shown to satisfy the condition $\mathbb{E}\left(e^{\epsilon R_{j}(t)}\right)<\infty$ for some $\epsilon>0$, and then to bound other reactions $R_{k}$ in terms of $R_{j}$, that is, to obtain an almost sure bound of the form

$$
R_{k}(t) \leq a+b R_{j}(t)
$$

The non-negativity of the species population $X(t)$ in a reaction networks implies linear inequality relations among the reaction counts $R_{j}(t)$ via

$$
X(t)=x_{0}+\nu R(t) \geq 0 .
$$

Thus we expect to bound some reactions in terms of the other. With this in mind, we define a reaction $j$ to be unconsuming if its associated stoichiometric vector $\nu_{j}$ is nonnegative: $\nu_{i j} \geq 0$ for $i=1, \ldots, n$. We shall call a reaction consuming if it is not unconsuming. We shall use $\mathcal{C}$ to denote the indices $j$ of all consuming reactions and use $\mathcal{U}$ to denote the indices $j$ of all unconsuming reactions.

We note that an unconsuming reaction may not be bounded above in terms of another reaction. Motivated by this fact, we introduce the following useful property regarding the unconsuming reactions which may or may not hold in a given network.

Property 4.1. Given $t>0$, for any $j \in \mathcal{U}$, there exists $\epsilon>0$ such that

$$
\mathbb{E}\left(e^{\epsilon R_{j}(t, c)}\right)<\infty .
$$


Remark 3. Property 4.1 is readily satisfied if all the propensities of the unconsuming reactions are bounded on $\mathcal{S}_{x_{0}}$. To see this, suppose that there exists $K>0$ such that the propensity $a_{j}(x, c)$ satisfies

$$
a_{j}(x, c) \leq K \quad \forall x \in \mathcal{S}_{x_{0}}
$$

Then $\mathbb{E}\left(e^{\epsilon R_{j}(t, c)}\right)<\infty$ for every $\epsilon>0$. By the random time change representation,

$$
R_{j}(t, c)=Y_{j}\left(\int_{0}^{t} a_{j}(X(s, c), c) d s\right) \leq Y_{j}(K t)
$$

Thus $R_{j}(t, c) \leq Y_{j}(K t)$ and consequently

$$
\mathbb{E}\left(e^{\epsilon R_{j}(t, c)}\right) \leq \mathbb{E}\left(e^{\epsilon Y_{j}(K t)}\right) .
$$

The result follows from the fact that $\mathbb{E}\left(e^{\epsilon Y_{j}(K t)}\right)<\infty$ for every $\epsilon>0$.

We note that, if all the unconsuming reactions are of the form $\emptyset \rightarrow S_{i}$ or $S_{j} \rightarrow S_{j}+S_{i}$ where the copy number of species $S_{j}$ is bounded on $\mathcal{S}_{x_{0}}$, then Remark 3 readily applies.

Without loss of generality, we are interested in the parameter $c=c_{1}$ at a nominal value $c^{*}>0$, i.e., the parameter of reaction 1 . If reaction $1 \in \mathcal{U}$, i.e., reaction 1 is unconsuming, then the required bound (23) is automatically satisfied if Property 4.1 holds. If reaction $1 \in \mathcal{C}$ and its propensity is unbounded on $\mathcal{S}_{x_{0}}$, it may yet be possible to bound $R_{1}(t)$ above in terms of the unconsuming reactions and furthermore if Property 4.1 holds, then (23) holds.

Example 4.2. As a motivating example, let us consider the chemical kinetics example with two species and three reactions:

$$
S_{1} \rightarrow S_{2}, \quad S_{1}+S_{2} \rightarrow \emptyset, \quad \emptyset \rightarrow S_{1}
$$

with

$$
\nu_{1}=(-1,1)^{\mathrm{T}}, \quad \nu_{2}=(-1,-1)^{\mathrm{T}}, \quad \nu_{3}=(1,0)^{\mathrm{T}} .
$$

The propensities are given by $a_{1}(x)=c_{1} x_{1}, a_{2}(x)=c_{2} x_{1} x_{2}$ and $a_{3}(x)=c_{3}$. We note that $\mathcal{S}_{x_{0}}$ is unbounded. Since the propensity of reaction channel 3 is constant, by Remark 3, there exists $\epsilon>0$ such that

$$
\mathbb{E}\left(e^{\epsilon R_{3}(t)}\right)<\infty
$$

Thus (23) holds when the parameter of interest is $c_{3}$. However, the other two reaction channels have unbounded propensities.

In order to bound $R_{1}(t)$ and $R_{2}(t)$ in terms of $R_{3}(t)$, we make explicit use of the fact that species population process $X(t)$ remains nonnegative. The process at any time $t \geq 0$ satisfies (4)

$$
X(t)=x_{0}+\nu R(t)
$$


and since $X(t) \geq 0$ we have that $x_{0}+\nu R(t) \geq 0$. We readily see that

$$
R_{1}(t) \leq R_{1}(t)+R_{2}(t) \leq x_{0,1}+R_{3}(t)
$$

Now (27) readily implies that

$$
\mathbb{E}\left(e^{\epsilon R_{i}(t)}\right)<\infty
$$

for $i=1,2$, showing the condition (23) with respect to all three parameters.

This example suggests the possibility that the relation $x_{0}+\nu R(t) \geq 0$ may imply that $R_{1}(t)$ (the reaction channel of interest) is bounded above in terms of a positive affine combination of those unconsuming reactions which satisfy Property 4.1. In general, this determination could be made as follows.

Suppose that $1 \in \mathcal{C}$. The fact that $X(t) \geq 0$ can be expressed by

$$
x_{0}+\sum_{j \in \mathcal{C}} \nu_{j} R_{j}(t)+\sum_{j \in \mathcal{U}} \nu_{j} R_{j}(t) \geq 0
$$

For $j \in \mathcal{U}$ let $\mu_{j} \in \mathbb{R}^{n}$ be defined by $\left(\mu_{j}\right)_{i}=\max \left\{0, \nu_{i, j}\right\}$. Then $X(t) \geq 0$ implies

$$
x_{0}+\sum_{j \in \mathcal{C}} \nu_{j} R_{j}(t)+\sum_{j \in \mathcal{U}} \mu_{j} R_{j}(t) \geq 0 .
$$

Letting

$$
y=x_{0}+\sum_{j \in \mathcal{U}} \mu_{j} R_{j}(t)
$$

and noting that $y \geq x_{0}$, motivates the linear programming (feasibility) problem:

$$
y+\sum_{j \in \mathcal{C}} \nu_{j} \xi_{j} \geq 0, \quad \xi \geq 0
$$

where $\xi \in \mathbb{R}^{n}$. The feasible region for $\xi$ is given by a convex polytope $\mathcal{R}_{y}$ which may be unbounded. If $\mathcal{R}_{y}$ is bounded in the $\xi_{1}$ direction, then one can obtain an upper bound for $R_{1}(t)$ as an affine combination of $R_{j}(t)$ for $j \in \mathcal{U}$. We note that whether $\mathcal{R}_{y}$ is bounded in the $\xi_{1}$ direction or not depends only on $\nu_{j}$ for $j \in \mathcal{C}$ and not on $y$.

Then Property 4.1 can be used to obtain (23). We summarize this discussion as a theorem.

Theorem 4.3. Given a non-explosive chemical reaction network with product form propensity functions, suppose that Property 4.1 holds and that either reaction 1 is unconsuming or the feasible region of the linear program (28) is bounded in the first coordinate $\xi_{1}$. Then (23) holds.

We illustrate the application of Theorem 4.3 by examining the following network that models gene expression. 
Example 4.4. Let us consider the following system of gene expression

$$
\begin{array}{ll}
R_{1}: A \stackrel{c_{1}}{\rightarrow} A+S_{1}, & R_{2}: A^{\prime} \stackrel{c_{2}}{\rightarrow} A^{\prime}+S_{1}, \quad R_{3}: S_{1} \stackrel{c_{3}}{\rightarrow} S_{2} \\
R_{4}: A+S_{2} \stackrel{\alpha}{\rightarrow} A^{\prime}, & R_{5}: A^{\prime} \stackrel{\beta}{\rightarrow} A+S_{2}
\end{array}
$$

with stoichiometric vectors

$$
\begin{aligned}
& \nu_{1}=(0,0,1,0)^{\mathrm{T}}, \quad \nu_{2}=(0,0,1,0)^{\mathrm{T}}, \quad \nu_{3}=(0,0,-1,1)^{\mathrm{T}}, \\
& \nu_{4}=(-1,1,0,-1)^{\mathrm{T}}, \quad \nu_{5}=(1,-1,0,1)^{\mathrm{T}} .
\end{aligned}
$$

Denote the population of $\left(A, A^{\prime}, S_{1}, S_{2}\right)$ by $\left(x_{1}, \ldots, x_{4}\right)$ and hence the propensities are

$$
a_{1}(x, c)=c_{1} x_{1}, a_{2}(x, c)=c_{2} x_{2}, a_{3}(x, c)=c_{3} x_{3}, a_{4}(x, c)=\alpha x_{1} x_{4}, a_{5}(x, c)=\beta x_{2} .
$$

Note that the total population of $A$ and $A^{\prime}$ is preserved and hence $a_{1}, a_{2}$ and $a_{5}$ are bounded on $\mathcal{S}_{x_{0}}$. Therefore, condition (23) with respect to $c_{1}, c_{2}$ and $\beta$ by Remark 3 , The propensities associated with $R_{3}$ and $R_{4}$ are not bounded as the populations of $S_{1}$ and $S_{2}$ are unbounded. However, note that

$$
X_{1}(t)=x_{0,1}-R_{4}(t)+R_{5}(t) \geq 0
$$

and therefore

$$
\mathbb{E}\left(e^{\epsilon R_{4}(t)}\right) \leq \mathbb{E}\left(e^{\epsilon\left(x_{0,1}+R_{5}(t)\right)}\right)<\infty
$$

for some $\epsilon>0$ since reaction 5 has bounded propensity. Similarly, note that

$$
X_{3}(t)=x_{0,3}+R_{1}(t)+R_{2}(t)-R_{3}(t) \geq 0
$$

and therefore

$$
\mathbb{E}\left(e^{\epsilon R_{3}(t)}\right) \leq \mathbb{E}\left(e^{\epsilon\left(x_{0,3}+R_{1}(t)+R_{2}(t)\right)}\right)<\infty
$$

Hence, condition (23) holds with respect to $c_{3}$ and $\alpha$ as well.

We note that the usefulness of Theorem 4.3 for reactions that have unbounded propensities relies on the condition involving the linear programming problem (28). We illustrate an example where this condition fails. That is, it is not possible to bound a reaction with unbounded propensity in terms of a reaction with bounded propensity.

Example 4.5. Consider the reaction system

$$
R_{1}: \emptyset \rightarrow S_{1} \quad R_{2}: 2 S_{1} \rightarrow 2 S_{2} \quad R_{3}: 2 S_{2} \rightarrow 2 S_{1}
$$

with stochastic mass action propensities $a_{1}(x)=c_{1}, a_{2}(x)=c_{2} x_{1}\left(x_{1}-1\right) / 2$ and $a_{3}(x)=c_{3} x_{2}\left(x_{2}-1\right) / 2$. The stoichiometric vectors are

$$
\nu_{1}=(1,0)^{\mathrm{T}}, \quad \nu_{2}=(-2,2)^{\mathrm{T}}, \quad \nu_{3}=(2,-2)^{\mathrm{T}} .
$$


The propensity of the first reaction is bounded and hence the exponential integrability $\mathbb{E}\left(e^{\epsilon R_{1}(t)}\right)<\infty$ holds for each $\epsilon>0$. However, if we are interested in the sensitivities with respect to $c_{2}$ or $c_{3}$, then we wish to verify $\mathbb{E}\left(e^{\epsilon R_{j}(t)}\right)<\infty$ for $j=2$ or 3 . Since $\mathcal{S}_{x_{0}}$ is unbounded, the propensities $a_{2}$ and $a_{3}$ are unbounded. Thus, it will be desirable to bound $R_{2}(t)$ and/or $R_{3}(t)$ in terms of $R_{1}(t)$.

The non-negativity of states, $X_{1}(t) \geq 0$ and $X_{2}(t) \geq 0$, again yields

$$
\begin{aligned}
& X_{1}(t)=x_{0,1}+R_{1}(t)-2 R_{2}(t)+2 R_{3}(t) \geq 0, \\
& X_{2}(t)=x_{0,2}+2 R_{2}(t)-2 R_{3}(t) \geq 0 .
\end{aligned}
$$

These inequalities permit arbitrarily large values for $R_{2}(t)$ and $R_{3}(t)$ for a given value of $R_{1}(t)$. Thus, it is not possible to bound $R_{2}(t)$ or $R_{3}(t)$ in terms of $R_{1}(t)$. This will be reflected in the failure of the condition on the linear programming problem (after a reordering of reaction indices) stated in Theorem 4.3.

Next, we focus on the situation where the system has an unconsuming reaction with unbounded propensity. Even if we may bound all other reactions in terms of this unconsuming reaction, one still needs to verify the Property 4.1 for this unconsuming reaction. We describe a coupling strategy inspired by [1, 12] and is based on the RTC (2) that allows us to verify Property 4.1 for a class of networks where unconsuming reactions may have unbounded propensities. We motivate the strategy through two examples.

Example 4.6. We consider the reaction system

$$
R_{1}: S \stackrel{c_{1}}{\longrightarrow} 2 S, \quad R_{2}: 2 S \stackrel{c_{2}}{\longrightarrow} \emptyset
$$

with stoichiometric vectors $\nu_{1}=1, \nu_{2}=-2$ and initial population $x_{0}$. We take $a_{1}(x)=$ $c_{1} x_{1}$ and $a_{2}(x)=c_{2} x_{2}\left(x_{2}-1\right) / 2$.

We note that the propensities are both unbounded. Our first aim is to justify that $\mathbb{E}\left(e^{\epsilon R_{1}(t, c)}\right)<\infty$ for some $\epsilon>0$. Let us denote $X(t)$ the population of species $S$ in the above reaction system. In the meanwhile, we consider another reaction system with a single reaction channel

$$
\widetilde{R}_{1}: \widetilde{S} \stackrel{c_{1}}{\longrightarrow} 2 \widetilde{S}
$$

with the same initial population $x_{0}$ and denote the population of $\widetilde{S}$ by $\widetilde{X}(t)$. Note that by the random time change representation (2) we can couple the processes $X(t)$ and $\widetilde{X}(t)$ in the same probability space using the following coupling

$$
\begin{aligned}
X(t)=x_{0} & +Y_{1}\left(\int_{0}^{t} a_{1}(X(s)) \wedge a_{1}(\widetilde{X}(s)) d s\right) \\
& +Y_{2}\left(\int_{0}^{t} a_{1}(X(s))-a_{1}(X(s)) \wedge a_{1}(\widetilde{X}(s)) d s\right) \\
& -2 Y_{3}\left(\int_{0}^{t} a_{2}(X(s)) d s\right)
\end{aligned}
$$


and

$$
\begin{aligned}
\widetilde{X}(t)=x_{0} & +Y_{1}\left(\int_{0}^{t} a_{1}(X(s)) \wedge a_{1}(\widetilde{X}(s)) d s\right) \\
+ & Y_{2}\left(\int_{0}^{t} a_{1}(\widetilde{X}(s))-a_{1}(X(s)) \wedge a_{1}(\widetilde{X}(s)) d s\right),
\end{aligned}
$$

where $Y_{i}, i=1,2,3$ are independent unit rate Poisson process carried by $(\Omega, \mathcal{F}, \mathbb{P})$. We note that

$$
\begin{aligned}
R_{1}(t) & =Y_{1}\left(\int_{0}^{t} a_{1}(X(s)) \wedge a_{1}(\widetilde{X}(s)) d s\right) \\
& +Y_{2}\left(\int_{0}^{t} a_{1}(X(s))-a_{1}(X(s)) \wedge a_{1}(\tilde{X}(s)) d s\right)
\end{aligned}
$$

and

$$
\begin{aligned}
\widetilde{R}_{1}(t) & =Y_{1}\left(\int_{0}^{t} a_{1}(X(s)) \wedge a_{1}(\widetilde{X}(s)) d s\right) \\
& +Y_{2}\left(\int_{0}^{t} a_{1}(\widetilde{X}(s))-a_{1}(X(s)) \wedge a_{1}(\widetilde{X}(s)) d s\right) .
\end{aligned}
$$

We claim that for any $t>0, X(t) \leq \widetilde{X}(t)$ almost surely. Define $T_{n}$ the $n$-th jump time of the processes $X(t)$ and $\widetilde{X}(t)$ combined. Set $T_{0}=0$. We note that $X\left(T_{0}\right)=$ $\widetilde{X}\left(T_{0}\right)=x_{0}$. Suppose $X\left(T_{j}\right) \leq \widetilde{X}\left(T_{j}\right)$ for $j=0, \ldots, n-1$ for some $n$. Since $X(t)$ and $\widetilde{X}(t)$ are constant between two successive jumps, $X(t) \leq \widetilde{X}(t)$ for all $t<T_{n}$ and hence by monotonicity of $a_{1}$, we have $a_{1}(X(t)) \leq a_{1}(\widetilde{X}(t))$ for all $t<T_{n}$. Thus

$$
\int_{0}^{T_{n}}\left(a_{1}(X(s))-a_{1}(\widetilde{X}(s)) \wedge a_{1}(X(s)) d s=0 .\right.
$$

Hence

$$
X\left(T_{n}\right)=x_{0}+Y_{1}\left(\int_{0}^{T_{n}} a_{1}(X(s)) d s\right)+Y_{1}(0)-2 Y_{3}\left(\int_{0}^{T_{n}} a_{2}(X(s)) d s\right)
$$

and

$$
\tilde{X}\left(T_{n}\right)=x_{0}+Y_{1}\left(\int_{0}^{T_{n}} a_{1}(X(s)) d s\right)+Y_{2}\left(\int_{0}^{T_{n}} a_{1}(\tilde{X}(s))-a_{1}(X(s)) d s\right) .
$$

On account of the fact that a unit Poisson process starts at zero, $Y_{1}(0)=0$, and it is clear that $X\left(T_{n}\right) \leq \tilde{X}\left(T_{n}\right)$, completing the induction.

Now since $a_{1}(X(t)) \leq a_{1}(\widetilde{X}(t))$ for any $t>0$, we deduce from (29) and (30) that

$$
\left.Y_{1}\left(\int_{0}^{t} a_{1}(X(s))\right) d s\right)=R_{1}(t)
$$


and

$$
\left.Y_{1}\left(\int_{0}^{t} a_{1}(X(s))\right) d s\right)+Y_{2}\left(\int_{0}^{t} a_{1}(\widetilde{X}(s))-a_{1}(X(s)) d s\right)=\widetilde{R}_{1}(t)
$$

showing $R_{1}(t) \leq \widetilde{R}_{1}(t)$. This in turn implies that $\mathbb{E}\left(e^{\epsilon R_{1}(t)}\right) \leq \mathbb{E}\left(e^{\epsilon \widetilde{R}_{1}(t)}\right)$. Consequently, the desired exponential integrability of $R_{1}(t)$ follows from the fact that the reaction count $\widetilde{R}_{1}(t)$ of auxiliary system $S \rightarrow 2 S$ is a negative binomial process and hence there exists $\epsilon>0$ such that $\mathbb{E}\left(e^{\epsilon \widetilde{R}_{1}(t)}\right)<\infty$ (see Appendix $\mathbb{B}$ ). Finally, the exponential integrability (23) holds with respect to $c_{2}$ since

$$
X(t)=x_{0}+R_{1}(t)-2 R_{2}(t) \geq 0
$$

and hence $R_{2}(t)$ can be bounded in terms of $R_{1}(t)$ whose exponential integrability has already been established.

The next example extends the coupling argument further.

Example 4.7. Let us consider the two species Lotka-Volterra model [10], namely,

$$
\begin{array}{llll}
R_{1}: \emptyset \stackrel{\alpha_{1}}{\longrightarrow} S_{1}, & R_{2}: S_{1} \stackrel{\beta_{1}}{\longrightarrow} 2 S_{1}, & R_{3}: S_{1}+S_{2} \stackrel{\gamma_{12}}{\longrightarrow} S_{2}, & R_{4}: S_{1} \stackrel{\delta_{1}}{\longrightarrow} \emptyset \\
R_{5}: \emptyset \stackrel{\alpha_{2}}{\longrightarrow} S_{2}, & R_{6}: S_{2} \stackrel{\beta_{2}}{\longrightarrow} 2 S_{2}, & R_{7}: S_{2}+S_{1} \stackrel{\gamma_{21}}{\longrightarrow} S_{1}, & R_{8}: S_{2} \stackrel{\delta_{2}}{\longrightarrow} \emptyset
\end{array}
$$

whose stoichiometric vectors are

$$
\begin{aligned}
& \nu_{1}=(1,0)^{\mathrm{T}}, \quad \nu_{2}=(1,0)^{\mathrm{T}}, \quad \nu_{3}=(-1,0)^{\mathrm{T}}, \quad \nu_{4}=(-1,0)^{\mathrm{T}} \\
& \nu_{5}=(0,1)^{\mathrm{T}}, \quad \nu_{6}=(0,1)^{\mathrm{T}}, \quad \nu_{7}=(0,-1)^{\mathrm{T}}, \quad \nu_{8}=(0,-1)^{\mathrm{T}}
\end{aligned}
$$

Since the propensity of reaction channel 1 is constant, $\mathbb{E}\left(e^{\epsilon R_{1}(t)}\right)<\infty$. The same argument applies to reaction channel 5. To show that both $\mathbb{E}\left(e^{\epsilon R_{2}(t)}\right)$ and $\mathbb{E}\left(e^{\epsilon R_{6}(t)}\right)$ are finite (for some $\epsilon>0$ ), we couple the system with the following elementary system (with rate parameters $\alpha_{1}+\beta_{1}$ and $\alpha_{2}+\beta_{2}$ )

$$
\widetilde{R}_{1}: \widetilde{S}_{1} \stackrel{\alpha_{1}+\beta_{1}}{\longrightarrow} 2 \widetilde{S}_{1}, \quad \widetilde{R}_{2}: \widetilde{S}_{2} \stackrel{\alpha_{2}+\beta_{2}}{\longrightarrow} 2 \widetilde{S}_{2}
$$


through

$$
\begin{aligned}
& X_{1}(t)=x_{0,1}+Y_{1,1}\left(\int_{0}^{t} \alpha_{1} d s\right)+Y_{1,2}\left(\int_{0}^{t} \beta_{1}\left(X_{1}(s) \wedge \widetilde{X}_{1}(s)\right) d s\right) \\
&+ Y_{1,3}\left(\int_{0}^{t} \beta_{1}\left(X_{1}(s)-X_{1}(s) \wedge \widetilde{X}_{1}(s)\right) d s\right) \\
&-Y_{1,4}\left(\int_{0}^{t} \gamma_{12} X_{1}(s) X_{2}(s) d s\right)-Y_{1,5}\left(\int_{0}^{t} \delta_{1} X_{1}(s) d s\right), \\
& X_{2}(t)=x_{0,2}+Y_{2,1}\left(\int_{0}^{t} \alpha_{2} d s\right)+Y_{2,2}\left(\int_{0}^{t} \beta_{2}\left(X_{2}(s) \wedge \widetilde{X}_{2}(s)\right) d s\right) \\
&+Y_{2,3}\left(\int_{0}^{t} \beta_{2}\left(X_{2}(s)-X_{2}(s) \wedge \widetilde{X}_{2}(s)\right) d s\right) \\
&-Y_{2,4}\left(\int_{0}^{t} \gamma_{21} X_{2}(s) X_{1}(s) d s\right)-Y_{2,5}\left(\int_{0}^{t} \delta_{2} X_{2}(s) d s\right)
\end{aligned}
$$

and

$$
\begin{aligned}
\widetilde{X}_{1}(t)=\widetilde{x}_{0,1} & +Y_{1,1}\left(\int_{0}^{t} \alpha_{1} \widetilde{X}_{1}(s) d s\right)+Y_{1,2}\left(\int_{0}^{t} \beta_{1}\left(X_{1}(s) \wedge \widetilde{X}_{1}(s)\right) d s\right) \\
& +Y_{1,6}\left(\int_{0}^{t} \beta_{1}\left(\widetilde{X}_{1}(s)-X_{1}(s) \wedge \widetilde{X}_{1}(s)\right) d s\right) \\
\widetilde{X}_{2}(t)=\widetilde{x}_{0,2} & +Y_{2,1}\left(\int_{0}^{t} \alpha_{2} \widetilde{X}_{2}(s) d s\right)+Y_{2,2}\left(\int_{0}^{t} \beta_{2}\left(X_{2}(s) \wedge \widetilde{X}_{2}(s)\right) d s\right) \\
& +Y_{2,6}\left(\int_{0}^{t} \beta_{2}\left(\widetilde{X}_{2}(s)-X_{2}(s) \wedge \widetilde{X}_{2}(s)\right) d s\right)
\end{aligned}
$$

where $Y_{1, i}$ and $Y_{2, i}$ for $i=1, \ldots, 6$ are independent unit rate Poisson processes carried by $(\Omega, \mathcal{F}, \mathbb{P})$ and the initial population $\widetilde{x}_{0,1}=x_{0,1}+1$ and $\widetilde{x}_{0,2}=x_{0,2}+1$. We show that $\mathbb{E}\left(e^{\epsilon R_{2}(t)}\right)$ is finite for sufficiently small $\epsilon>0$. To this end, note that the reaction event counts $R_{1}(t), R_{2}(t)$ and $\widetilde{R}_{1}(t)$ are given by

$$
\begin{aligned}
R_{1}(t)= & Y_{1,1}\left(\int_{0}^{t} \alpha_{1} d s\right) \\
R_{2}(t)= & Y_{1,2}\left(\int_{0}^{t} \beta_{1}\left(X_{1}(s) \wedge \widetilde{X}_{1}(s)\right) d s\right)+Y_{1,3}\left(\int_{0}^{t} \beta_{1}\left(X_{1}(s)-X_{1}(s) \wedge \widetilde{X}_{1}(s)\right) d s\right), \\
\widetilde{R}_{1}(t)= & Y_{1,1}\left(\int_{0}^{t} \alpha_{1} \widetilde{X}_{1}(s) d s\right)+Y_{1,2}\left(\int_{0}^{t} \beta_{1}\left(X_{1}(s) \wedge \widetilde{X}_{1}(s)\right) d s\right) \\
& +Y_{1,6}\left(\int_{0}^{t} \beta_{1}\left(\widetilde{X}_{1}(s)-X_{1}(s) \wedge \widetilde{X}_{1}(s)\right) d s\right) .
\end{aligned}
$$

We further note that $\widetilde{X}_{1}(t) \geq \widetilde{x}_{0,1} \geq 1$. Hence

$$
Y_{1,1}\left(\int_{0}^{t} \alpha_{1} d s\right) \leq Y_{1,1}\left(\int_{0}^{t} \alpha_{1} \widetilde{X}_{1}(s) d s\right) .
$$


We show that $X_{1}(t) \leq \widetilde{X}_{1}(t)$ for all $t \geq 0$ following a similar argument as in Example 4.6. Define the $n$th jump time of the combined process $\left(X_{1}(t), \widetilde{X}_{1}(t)\right)$ to be $T_{n}$ and set $T_{0}=0$. We observe $X_{1}\left(T_{0}\right) \leq \widetilde{X}_{1}\left(T_{0}\right)$. Suppose $X_{1}\left(T_{j}\right) \leq \widetilde{X}_{1}\left(T_{j}\right)$ for $j=0, \ldots, n-1$ for some $n$. Then, following an argument similar to that in Example 4.6, (and noting $\left.Y_{1,3}(0)=0\right)$ we obtain

$$
\begin{aligned}
X_{1}\left(T_{n}\right)=x_{0,1} & +Y_{1,1}\left(\int_{0}^{T_{n}} \alpha_{1} d s\right)+Y_{1,2}\left(\int_{0}^{T_{n}} \beta_{1} X_{1}(s) d s\right) \\
& -Y_{1,4}\left(\int_{0}^{T_{n}} \gamma_{12} X_{1}(s) X_{2}(s) d s\right)-Y_{1,5}\left(\int_{0}^{T_{n}} \delta_{1} X_{1}(s) d s\right)
\end{aligned}
$$

and

$$
\begin{aligned}
\widetilde{X}_{1}\left(T_{n}\right) & =\widetilde{x}_{0,1}+Y_{1,1}\left(\int_{0}^{T_{n}} \alpha_{1} \widetilde{X}_{1}(s) d s\right)+Y_{1,2}\left(\int_{0}^{T_{n}} \beta_{1} X_{1}(s) d s\right) \\
& +Y_{1,6}\left(\int_{0}^{t} \beta_{1}\left(\widetilde{X}_{1}(s)-X_{1}(s)\right) d s\right),
\end{aligned}
$$

which shows $X_{1}\left(T_{n}\right) \leq \widetilde{X}_{1}\left(T_{n}\right)$ completing the induction.

Since $X_{1}(t) \leq \widetilde{X}_{1}(t)$ and $\widetilde{X}_{1}(t) \geq 1$ for all $t \geq 0$, we obtain that

$$
\begin{aligned}
R_{1}(t)+R_{2}(t) & =Y_{1,1}\left(\int_{0}^{t} \alpha_{1} d s\right)+Y_{1,2}\left(\int_{0}^{t} \beta_{1} X_{1}(s) d s\right) \\
& \leq Y_{1,1}\left(\int_{0}^{t} \alpha_{1} \widetilde{X}_{1}(s) d s\right)+Y_{1,2}\left(\int_{0}^{t} \beta_{1} X_{1}(s) d s\right) \leq \widetilde{X}_{1}(t)
\end{aligned}
$$

for all $t \geq 0$. Since $\widetilde{X}_{1}(t)$ follows the negative binomial distribution, there exists $\epsilon>0$ such that

$$
\mathbb{E}\left(e^{\epsilon\left(R_{1}(t)+R_{2}(t)\right)}\right) \leq \mathbb{E}\left(e^{\epsilon \widetilde{X}_{1}(t)}\right)<\infty
$$

Applying the same argument to $R_{5}(t), R_{6}(t)$ and $\widetilde{R}_{2}(t)$ leads to

$$
\mathbb{E}\left(e^{\epsilon\left(R_{5}(t)+R_{6}(t)\right)}\right) \leq \mathbb{E}\left(e^{\epsilon \tilde{X}_{2}(t)}\right)<\infty
$$

since $\widetilde{X}_{2}(t)$ is negative binomially distributed as well.

Finally, for sensitivities with respect to $\gamma_{12}\left(\gamma_{21}\right)$ or $\delta_{1}\left(\delta_{2}\right)$, note that

$$
\begin{aligned}
& X_{1}(t)=x_{0,1}+R_{1}(t)+R_{2}(t)-R_{3}(t)-R_{4}(t) \geq 0, \\
& X_{2}(t)=x_{0,2}+R_{5}(t)+R_{6}(t)-R_{7}(t)-R_{8}(t) \geq 0,
\end{aligned}
$$

and hence $R_{3}(t)+R_{4}(t)$ and $R_{5}(t)+R_{6}(t)$ can be bounded by $x_{0,1}+R_{1}(t)+R_{2}(t)$ and $x_{0,2}+R_{5}(t)+R_{6}(t)$, respectively. Their exponential integrability follows directly from (31) and (32).

Examples 4.6 and 4.7 suggest a general result for verifying Property 4.1 for a class of reaction networks. We summarize the result in the following theorem. 
Theorem 4.8. Given a non-explosive chemical reaction network with product form propensity functions, we consider a class of systems that satisfies the following conditions.

(1) All unconsuming reactions have the form $A \rightarrow A+S_{i}$ where $S_{i}$ is a species and $A$ is a species (or empty) with bounded population (we refer to this as type 1 reaction) or have the form $S_{i} \rightarrow 2 S_{i}$ where $S_{i}$ is a species (we refer to the this as type 2 reaction).

(2) Let $S_{1}, S_{2}, . ., S_{k}$ be the set of species involved in type 2 unconsuming reactions in the previous condition. Then none of the consuming reactions result in an increase of any of the species $S_{i}$ for $i=1, . ., k$.

Then Property 4.1 is satisfied.

Remark 4. Some remarks are in order.

- The coupling strategy can definitely be applied to other classes of system given that we can identify an elementary subsystem which satisfies Property 4.1 in order to bound the growth of reaction counts of the full system.

- The above result does not apply to the example such as

$$
S_{1} \rightarrow 2 S_{1}, \quad S_{1}+S_{2} \rightarrow 2 S_{1}, \quad 2 S_{1} \rightarrow S_{1}+S_{2},
$$

where the consuming reaction $S_{1}+S_{2} \rightarrow 2 S_{1}$ increases the population of $S_{1}$. In this case, coupling the system with $\widetilde{S}_{1} \rightarrow 2 \widetilde{S}_{1}$ does not work because we can not bound the population of $S_{1}$ by that of $\widetilde{S}_{1}$. Verifying Property 4.1 for this type of examples will be the focus of another work.

\section{References}

[1] D.F. Anderson, An efficient finite difference method for parameter sensitivities of continuous time Markov chains, SIAM J. Numer. Anal. 50 (2012), pp. 2237-2258.

[2] S. Asmussen and P.W. Glynn, Stochastic simulation: algorithms and analysis, Vol. 57, Springer Science \& Business Media, 2007.

[3] P. Brémaud, Point processes and queues: martingale dynamics, Vol. 50, Springer, 1981.

[4] S. Engblom, On the stability of stochastic jump kinetics, Applied Mathematics 5 (2014), pp. 3217-3239.

[5] S.N. Ethier and T.G. Kurtz, Markov Processes: Characterization and Convergence, 2nd ed., John Wiley \& Sons, Inc., New York, 2005.

[6] D.T. Gillespie, Exact stochastic simulation of coupled chemical reactions, J. Phys. Chem. 81 (1977), pp. 2340-2361.

[7] P.W. Glynn and P. L'ecuyer, Likelihood ratio gradient estimation for stochastic recursions, Advances in applied probability 27 (1995), pp. 1019-1053.

[8] A. Gupta and M. Khammash, Unbiased estimation of parameter sensitivities for stochastic chemical reaction networks, SIAM J. Sci. Comput. 35 (2013), pp. A2598-A2620.

[9] A. Gupta and M. Khammash, An efficient and unbiased method for sensitivity analysis of stochastic reaction networks, J. R. Soc. Interface (2014), p. 20140979.

[10] A. Gupta, C. Briat, and M. Khammash, A scalable computational framework for establishing long-term behavior of stochastic reaction networks, PLoS computational biology 10 (2014), p. e1003669.

[11] T. Jahnke and W. Huisinga, Solving the chemical master equation for monomolecular reaction systems analytically, Journal of mathematical biology 54 (2007), pp. 1-26. 
[12] T.G. Kurtz, Representation and approximation of counting processes, in Advances in filtering and optimal stochastic control, Springer, 1982, pp. 177-191.

[13] P. L'Ecuyer, Note: On the interchange of derivative and expectation for likelihood ratio derivative estimators, Management Science 41 (1995), pp. 738-747.

[14] S. Plyasunov and A.P. Arkin., Efficient stochastic sensitivity analysis of discrete event systems, J. Comput. Phys. 221 (2007), pp. 724-738.

[15] P.E. Protter, Stochastic differential equations, Springer, 2005.

[16] M. Rathinam, P.W. Sheppard, and M. Khammash, Efficient computation of parameter sensitivities of discrete stochastic chemical reaction networks, J. Chem. Phys. 132 (2010), p. 034103.

[17] M. Rathinam, Moment growth bounds on continuous time markov processes on nonnegative integer lattices, Quart. Appl. Math. 53 (2015), pp. 347-364.

[18] P.W. Sheppard, M. Rathinam, and M. Khammash, A pathwise derivative approach to the computation of parameter sensitivities in discrete stochastic chemical systems, J. Chem. Phys. 136 (2012), p. 034115.

[19] T. Wang and M. Rathinam, Efficiency of the Girsanov transformation approach for parametric sensitivity analysis of stochastic chemical kinetics, JUQ 4 (2016), pp. 1288-1322.

\section{Appendix A. Differentiating Inside an Integral}

Theorem A.1. (Asmussen \& Glynn) [2]) Suppose $G(c, \omega)$ is a random variable for each $c$ in some interval of the real line. Let $c_{r e f}$ be a specific value of $c$. Suppose the following hold:

(1) For a set of $\omega$ with probability one, $G(c, \omega)$ is differentiable with respect to $c$ at $c=c_{\text {ref }}$.

(2) There exists an interval $\left(c_{l}, c_{u}\right)$ containing $c_{r e f}$ (independent of $\omega$ ) on which $G(c, \omega)$ is Lipschitz (in c) for a set of $\omega$ with probability one, with constant $K$ which may depend on $\omega$. That is, for any $c_{1}, c_{2}$ in the interval $\left(c_{l}, c_{u}\right)$, the following holds:

$$
\left|G\left(c_{1}, \omega\right)-G\left(c_{2}, \omega\right)\right| \leq K(\omega)\left|c_{1}-c_{2}\right|
$$

(3) $\mathbb{E}(K)$ is finite.

(4) $\mathbb{E}(|G(c, \omega)|)$ is finite for all $c$ in $\left(c_{l}, c_{u}\right)$.

Then the following holds:

$$
\left.\frac{d}{d c}\right|_{c=c_{r e f}} \mathbb{E}(G(c))=\mathbb{E}\left(\left.\frac{d}{d c}\right|_{c=c_{r e f}} G(c)\right) .
$$

\section{Appendix B. Probability mass function of the pure birth process $S \rightarrow 2 S$}

We consider reaction system consisting of single species $S$ and single reaction channel $S \rightarrow 2 S$. The species count process $X(t)$ evolves on state space is $\mathbb{Z}_{+}$. Let the reaction propensity be given by $c x$. That is given $X(t)=x$, the probability of one reaction event during $(t, t+h]$ is exactly $c x h+o(h)$ as $h \rightarrow 0+$. We suppose that $X(0)=x$ with probability one. 
Define $p_{n}(t)=\operatorname{Prob}(X(t)=n)$ for $n \in \mathbb{Z}_{+}$. The Kolmogorov's forward equations are given by

$$
\begin{aligned}
& p_{n}^{\prime}(t)=-c n p_{n}(t)+c(n-1) p_{n-1}(t) \quad n \geq x+1 \\
& p_{x}^{\prime}(t)=-\operatorname{cxp}_{x}(t)
\end{aligned}
$$

We note that $p_{n}(t)=0$ for all $n<x$ and $t \geq 0$. Also the initial conditions are $p_{x}(0)=1$ and $p_{n}(0)=0$ for $n \neq x$.

For $x \geq 1$, we claim that the solution is given by

$$
p_{x+k}(t)=\frac{(x+k-1) !}{k !(x-1) !} q^{x} p^{k} \quad k \geq 0
$$

where $q=e^{-c t}$ and $p=1-q$. We note that when $k=0$, this gives $p_{x}(t)=e^{-c x t}$. Thus the probability mass function follows a negative binomial distribution.

Clearly, the formula for $p_{x}(t)$ is correct. To verify the correctness of $p_{x+k}(t)$ for $k \geq 1$, we simply verify the forward equations. We first note that $q^{\prime}=-c q$ and $p^{\prime}=c q$. We obtain that

$$
p_{x+k}^{\prime}(t)=-\frac{(x+k-1) !}{k !(x-1) !} c x q^{x} p^{k}+\frac{(x+k-1) !}{k !(x-1) !} c k q^{x+1} p^{k-1} .
$$

Hence

$$
\begin{aligned}
p_{x+k}^{\prime}+c(x+k) p_{x+k} & =\frac{(x+k-1) !}{k !(x-1) !} c k q^{x} p^{k}+\frac{(x+k-1) !}{k !(x-1) !} c k q^{x+1} p^{k-1} \\
& =c k \frac{(x+k-1) !}{k !(x-1) !} q^{x} p^{k-1}(q+p)=c(x+k-1) p_{x+k-1},
\end{aligned}
$$

which verifies the forward equations.

We show that for each $t>0$, there exists $\epsilon>0$ such that $\mathbb{E}\left(e^{\epsilon X(t)}\right)<\infty$. In fact

$$
\mathbb{E}\left(e^{\epsilon X(t)}\right)=\sum_{k=0}^{\infty} e^{\epsilon(x+k)} p_{x+k}(t)
$$

To show that the sum is finite, we use the ratio test. The ratio of the $k+1$ st term to the $k$-th term

$$
M_{k+1} / M_{k}=e^{\epsilon x} \frac{x+k}{k+1}\left(1-e^{-c t}\right)
$$

which limits to $e^{\epsilon x}\left(1-e^{-c t}\right)$ as $k \rightarrow \infty$ and the limit is less than 1 for sufficiently small $\epsilon>0$. 